\title{
Molecular technology vs clinical practice: a hypothesis in genetics
}

\author{
Journal of Human Genetics (2010) 55, 552-553; doi:10.1038/jhg.2010.61; published online 3 June 2010
}

Medical genetics is the application of genetics to the study of human health and diseases. Typically, a medical geneticist is a highly trained research laboratory professional who can additionally take on the role of a consultant to physicians. A clinical geneticist is a physician involved in all parts of clinical practice related to genetic disorders. This role is becoming increasingly important as society strives to deal with the tremendous explosion of genetic information arising as a consequence of the Human Genome and the Human Variome Projects. The excitement of the specialty lies in the clinician understanding the evolving human gene map and rapid technological advances, and then using these together with clinical skills and knowledge of inheritance and genetic mechanisms for the benefit of the patients and their families. But does the explosive evolution of technology become a useful tool in the hands of the clinical geneticist, or is it capable of dislodging the specialty?

The number of persons with internet access worldwide has been increasing dramatically over the previous years, making mutation databases accessible and genetic information freely available through forums to more people every day. It is probable that the genetic counseling services could be offered either by the internet or by software programs, giving rise to issues of interpretation. Another issue that weakens clinical genetics as an entity is that it is not recognized as an official specialty in several countries worldwide. As a result, genetic counseling is exercised by professionals with a relevant background, physicians of different specialties or biologists, including cytogeneticists and molecular biologists.

An impressive progression in technology over the last decades led us from old-fashioned cytogenetics to modern molecular genetics and molecular cytogenetics. Nextgeneration sequencing and genotyping technologies will make even larger amounts of personal genetic information readily available at ever-diminishing costs. ${ }^{1}$ It can be argued that although some of this information will be about known variants and could influence the management of patients, most will be of no direct medical value. However, recent advances in high-throughput DNA sequencing technologies have shown that singlemolecule sequencing-enabled analysis of human genomic information without the need for cloning, amplification or ligation is now a fact. ${ }^{2}$ The revolution of these technologies has now made it feasible to determine the genome sequences of many individuals. Using current approaches, whole human genome sequences are not typically assembled and determined de novo, but instead, variations relative to a reference sequence are identified. The main steps involved in determining a genome sequence include single-nucleotide polymorphisms and structural variations, assembling new sequences, and phasing haplotypes. ${ }^{3}$

Lately, there has been an ongoing debate regarding direct-to-consumer genetic tests, which have introduced a new trend, personal genomics. Discussions relate to the evaluation of the utility of personal genomic information and include social, economical and ethical issues. It is apparent that the launch of this trend was triggered by new technologies that offer the potential for revolutionary changes in the practice of medicine from molecular diagnostic tests that detect disease before symptoms are evident to patient profiling techniques that help predict which patients are most likely to benefit from or be harmed by specific therapies. ${ }^{4}$ Personal genome information presents the additional complication of returning very large amounts of data about variants that will range in precision from complete penetrance to completely unknown significance, making the usual criterion of 'clinical validity' less straightforward as a measure of utility. Some variants found in the genome of an individual will have clinical validity, whereas most will not. Alternatively, the accuracy and transparency of the interpretations made of the potential significance of the full range of an individual's personal genomic information may become a key criterion for evaluating its utility for anticipating health risks. The quality of such interpretations will depend on how increasing knowledge is systematically used to neither understand nor overstate the constellation of risks that are encoded in individual genomes. ${ }^{5}$

If this trend prevails, and given that molecular techniques lead to a dramatic accumulation of information, it might be probable that in time molecular genetics will dominate not only neonatal screening or personal genomics, but also prenatal diagnosis. If we assume that such applications would become a routine for prenatal diagnosis in the future, and that we will be able to accurately determine the pathogenicity of any genomic variant in any individual, then the conclusion that genetic diseases will gradually diminish might be more than a speculation. Ethicists and others have complained that bringing genomic services to the market quickly is out of sequence with normal translational practice and should be a cause of concern. ${ }^{6}$ The rapid expansion of molecular genetics, due to the explosive evolution of molecular technology and the declining costs of wholegenome analyses, can progressively result in even larger segments of the human population having access to their personal genomes. Furthermore, a total invasion of these techniques in prenatal diagnosis in the (very far?) future would cause severe reduction in births 
of individuals with genetic diseases, displacing clinical genetics.

This commentary is the outcome of discussions between colleagues, both molecular and clinical geneticists, all working harmoniously to serve a common cause, the good of public health. It is not in any way endorsed by the author and will have served its purpose well if it becomes a point of fruitful discussion among professionals related to medical genetics.
Haris Kokotas

Department of Genetics, Institute of Child Health, 'Aghia Sophia' Children's Hospital,

Athens, Greece

E-mail: hkokotas@ich.gr

1 Mardis, E. R. The impact of next-generation sequencing technology on genetics. Trends Genet. 24, 133-141 (2008)
2 Pushkarev, D., Neff, N. F. \& Quake, S. R. Single-molecule sequencing of an individual human genome. Nat. Biotechnol. 27, 847-852 (2009).

3 Snyder, M., Du, J. \& Gerstein, M. Personal genome sequencing: current approaches and challenges. Genes Dev. 24, 423-431 (2010).

4 Schulman, K. A., Vidal, A. V. \& Ackerly, D. C. Personalized medicine and disruptive innovation: implications for technology assessment. Genet. Med. 11, 577-581 (2009).

5 Foster, M. W., Mulvihill, J. J. \& Sharp, R. R. Evaluating the utility of personal genomic information. Genet. Med. 11, 570-574 (2009).

6 Foster, M. W. \& Sharp, R. R. Out of sequence: how consumer genomics could displace clinical genetics. Nat. Rev. Genet. 9, 419 (2008). 